\title{
Supporting Sit-To-Stand Rehabilitation Using Smartphone Sensors and Arduino Haptic Feedback Modules
}

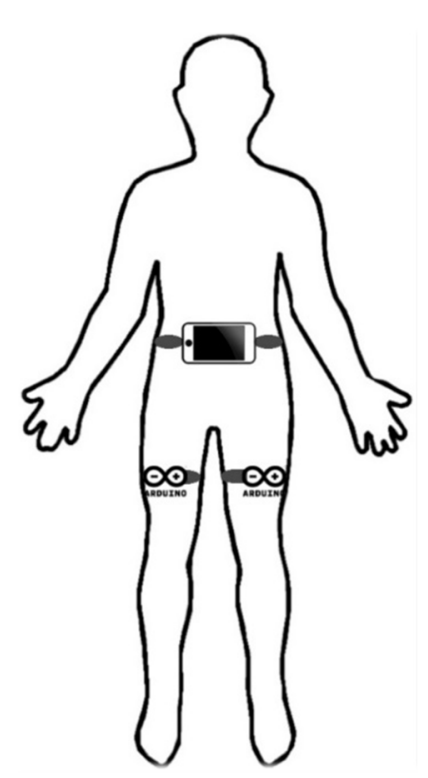

Figure 1: Outline design of hardware on body

\section{Craig O'Neil}

Computer and Information Sciences

University of Strathclyde

Glasgow G12 8QQ, Scotland, UK

craigo1409@gmail.com

\section{Mark D Dunlop}

Computer and Information Sciences

University of Strathclyde

Glasgow G12 8QQ, Scotland, UK

mark.dunlop@strath.ac.uk

\section{Andrew Kerr}

Biomedical Engineering

University of Strathclyde

Glasgow G12 800, Scotland, UK

a.kerr@strath.ac.uk

Permission to make digital or hard copies of part or all of this work for personal or classroom use is granted without fee provided that copies are not made or distributed for profit or commercial advantage and that copies bear this notice and the full citation on the first page. Copyright copies bear this notice and the full citation on the first page. Copyrights
for third-party components of this work must be honored. For all other uses, contact the Owner/Author.

Copyright is held by the owner/author(s).

MobileHCI '15 Adjunct, August 25-28, 2015, Copenhagen, Denmark

ACM 978-1-4503-3653-6/15/08.

http://dx.doi.org/10.1145/2786567.2793705

\begin{abstract}
The aim of this project is to design and build a system to aid patients in their rehabilitation after suffering a stroke. A stroke is one of the most serious conditions that an individual can suffer from, and the rehabilitation is often a long and difficult process. For many with movement effects, the sit-to-stand exercise is an important step in rehabilitation. The focus of this ongoing project is to create a system to assist sit-to-stand rehabilitation through the use of haptic feedback on balance. In this poster we present our initial prototype using standard smartphone accelerometers linked wirelessly to Arduino based vibration feedback modules mounted on the patients' legs. Initial feedback on the prototype is promising.
\end{abstract}

\section{Author Keywords}

Haptic feedback; Smartphone; Rehabilitation; Exercise

\section{ACM Classification Keywords}

H.5.2 Information Interfaces and Presentation: User Interfaces - Haptic I/O

\section{Introduction}

Motor impairments have been shown to affect about $80 \%$ of patients after a stroke [5]. Such impairments 


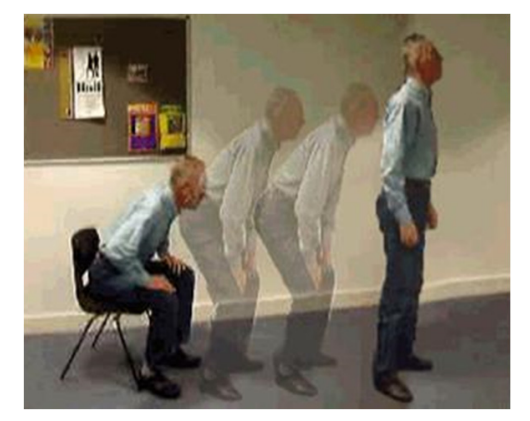

Figure 2: Sample sit-to-stand exercise can be caused by either ischemic or hemorrhagic injury to any of the motor cortex, premotor cortex, motor tracts or associated pathways between these areas in the brain. Research has shown that steady practice can help to rebuild these connections and improve quality of life for patients. However, rehabilitation exercises are often repetitive and boring for patients with little direct feedback on the quality of their efforts.

Visual feedback and gamification has been shown to give useful feedback for rehabilitation (e.g. [10]) while tactile feedback has been shown to have strong benefits for physical activity instruction (e.g. [9]). In our research we aim to combine these two elements to give tactile feedback directly to affected limbs using vibration modules linked wirelessly to a chest worn standard smartphone. Our long-term goal is to develop games and feedback mechanisms to encourage and support repetitive rehabilitation, particularly in this project assisting sit-to-stand exercise as these are a key stage of rehabilitation for many (e.g. Figure 2). In this poster we present our initial prototype and feedback.

\section{Background}

The main motivation for undertaking this project is to provide a new solution to one of the main problems that patients face in their rehabilitation after suffering a stroke, motor impairment.

One of the main reasons that has been found that prevents patients from completing regular rehabilitative practice is a lack of motivation on behalf of the patient [11]. Most exercises that are currently prescribed for home rehabilitation are very mundane and provide the patient with no feedback on how they are progressing.
This causes patients motivation levels to drop as they can often feel that no progress is being made. Another key problem that is faced by patients in rehabilitation is that often many of the exercises prescribed to them are impossible to do at home when recovering, particularly if living alone [11]. This is because many of the rehabilitation programs involve high-tech equipment, as well as often requiring specialists to operate. Another reason that training at home can be difficult is that often a physician is required in order to assist with training and judge how effective the training is progressing [4]. This means that in many situations patients are only able to train occasionally, and thus the rehabilitation is not as effective as it could have been had there been a system available that would allow patients to effectively continue their rehabilitation in their own time at home.

Tactile feedback has been shown to be beneficial when learning motor skills, and can help to instruct a user on both how and when to perform a movement, as well as providing the user with feedback when a movement is incorrect [8]. In the study by Spelmezan, it was shown that motor skills can be improved by using tactile feedback through the use of vibration motors whilst practicing snowboarding. This result has built upon research in a number of different sports in helping to improve motor skills, e.g. dancing [6], rowing [3] and karate [2]. Although, these are vastly different areas from stroke rehabilitation, the principle is very similar: The use of feedback helped the users to understand better what they were doing correctly or incorrectly, and how they should adapt in order to improve. This could tackle one of the main struggles, as mentioned above, that many stroke rehabilitation patients simply 


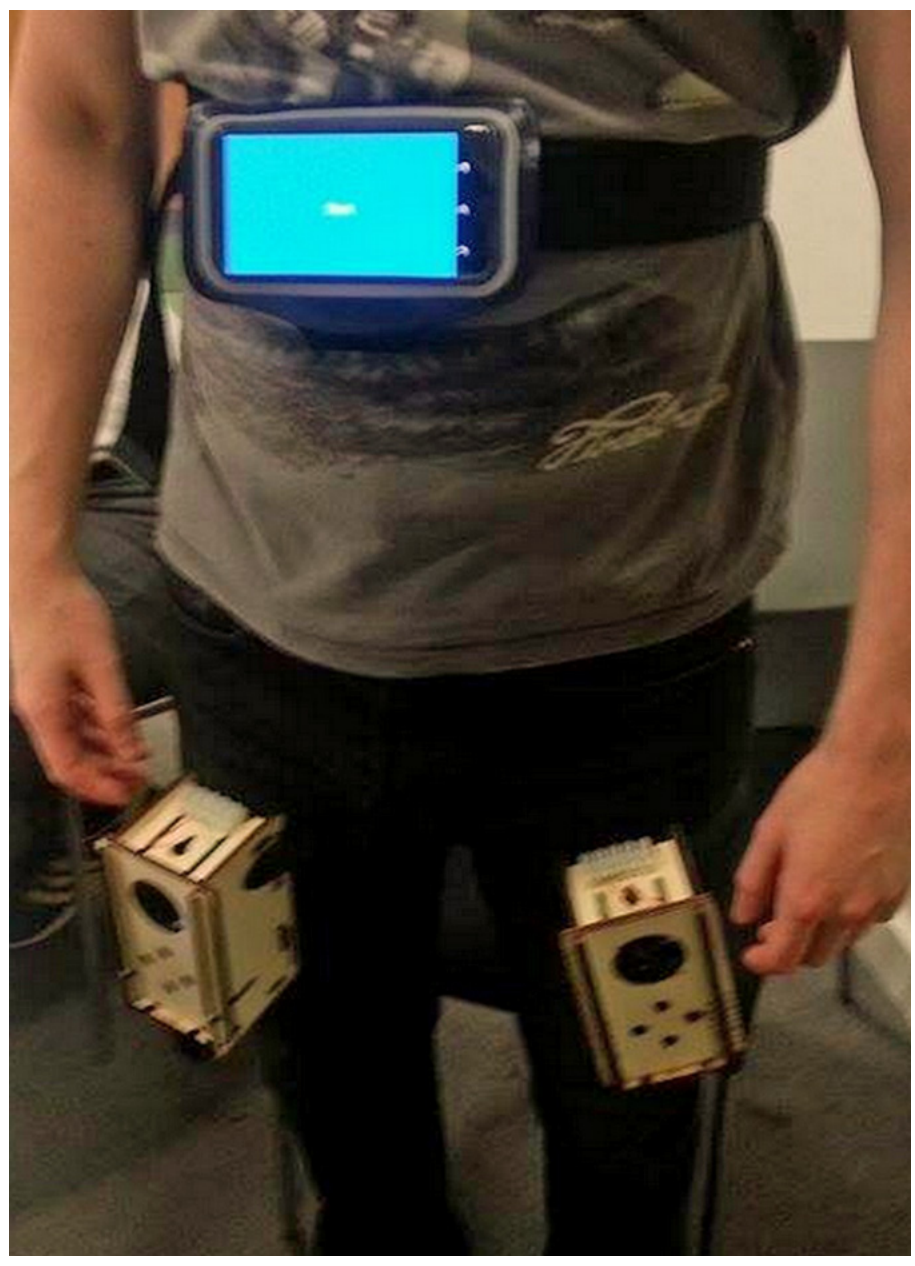

Figure 3: Prototype system showing smartphone on chest mounts and two wireless leg feedback modules don't understand if they are improving or not when practicing without the aid of a specialist.

A more recent study which shows the benefits of tactile feedback to motor skill learning is that of Bark et al. who conducted an investigation into the effects of Vibrotactile feedback on human learning of arm motions [1]. They developed a system that utilized vibrating actuators attached to an arm strap to send vibrations to different areas of the arm in order to tell the wearer how to correct their movements. The movements that the user's had to perform was to mimic a motion displayed on a screen in front of them. The accuracy of each movement was decided by software that utilized a Microsoft Kinect camera to track the users movement, and then decide from this if the movement is correct or not, dependent on the movement that they are aiming for. This allowed the software to calculate if the movement was correct, and if it was not, send vibrations to the arm in order to help correct the movement. The aim of this was to help improve the user's ability to learn new motor skills when moving their arm. The results of this study showed that the use of tactile feedback was beneficial in helping the users to learn new motor skills, reinforcing the findings previously reported by Spelmezan. The report also discusses the possible benefits of using the system in the future to aid stroke patients through their rehabilitation. The study predicts that the system would be beneficial in helping patients to re-learn the motor skills that may have been affected by a stroke. This is the main aim of this project, and the positive results reported in this study show that it is an area with great potential. 


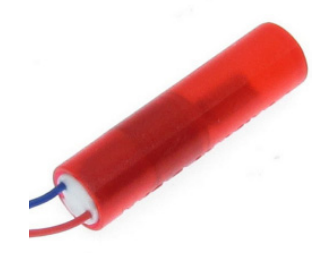

Figure 4: Precision Microdrive Pico Vibe $5 \mathrm{~mm}$ Vibration Motor (20 mm long, 5.3mm diam)
There is an ever growing range of mobile applications currently available aimed at improving health and fitness of users. A survey by research2guidance showed that the number of mobile health apps on the Apple Store and Google Play app stores surpassed 100,000 in the first quarter of 2014, more than doubling the value recorded in 2011 [7]. This shows a rapid and steady rise in applications and shows that it is an ever growing market. Such applications are wide and varied, many of them are simply for tracking health related values such as weight or calorie intake, e.g. Calorie Counter by MyFitnessPal. Other apps link with 3rd party technologies in order to provide more advanced assistance with fitness, such as the Nike+ Running app. This includes motion tracking technologies allowing mobile phones to track the number of steps a user takes, or the number of exercises a user performs but none that provides haptic feedback for a stroke patient's recovery.

\section{System Design}

Lack of viable methods for exercising at home to aid rehabilitation is a major problem faced by stroke rehabilitation patients. Our aim is to create a system that will be capable of providing haptic feedback to a user when performing their sit-to-stand exercises as part of their rehabilitation - particularly focusing on improving their balance and symmetry of standing. We developed a prototype system using Arduino microcontrollers wireless connected to standard smartphone in order to give tactile feedback to the user's leg if they are leaning to side while standing based on the smartphone accelerometer readings.

\section{Hardware Design}

Our main goal is to support widespread use at home, thus a system based around a smartphone is ideal given the ever increasing number of people using smartphones. The choice of Arduino for the prototype was purely practical as the platform gives great flexibility during prototyping at a low cost and can be fully battery powered. Our prototype is composed of an Android smartphone connected to two leg modules wirelessly (see Figures 1 and 3). The leg modules are based on Arduino Unos, ATmega328 microcontroller boards with $32 \mathrm{kB}$ of flash memory each linked to Adafruit Motor shields to control vibration. Tactile feedback was given through Precision Microdrive Pico Vibe $5 \mathrm{~mm}$ Vibration Motors (Figure 4) that provided a high level of vibration, normalised amplitude of up to $0.95 \mathrm{G}$ perpendicular to the leg making it feel considerably stronger than alternatives with no overheating concerns. An Adafruit Bluetooth Shield was added to one board to communicate with the mobile phone while a pair of Xbee transceivers were used for communication between the two leg modules. This means that the mobile phone and Arduino can move freely without any wiring between them, allowing the user to move as naturally as possible without any real or perceived limitation due to wiring. The phone was attached to the chest using a modified chest strap and the feedback units to legs using Velcro straps on custom cut cases for the Arduino stacks with the motor mounted between leg and case. The final units were unfortunately quite bulky, especially once sufficient battery power was included in the cases. However, it was felt better to have bulky completely wireless prototypes than smaller wired modules. 


\section{Software Design}

To maximize platform independence the smartphone software was developed in HTML5 / JavaScript running within a small Android Java wrapper application to support Bluetooth communication.

The system has to be able to first read the initial orientations of the phone when attached to the user, in order to gain a base value for upright. On startup the
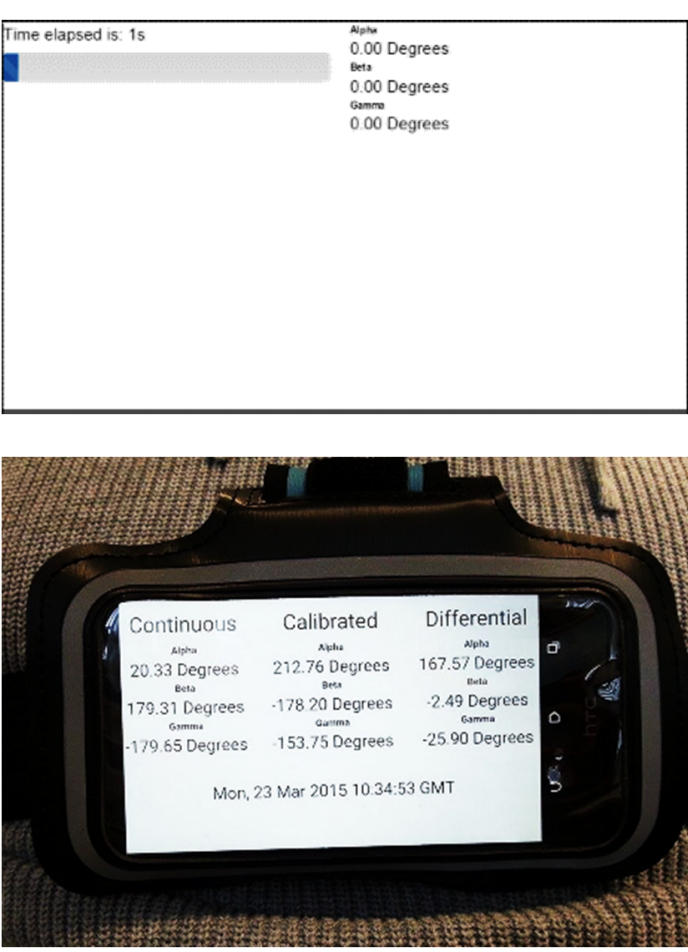

Figure 5: Calibration progress screen and sensing screen in phone mounting application connects with the master leg module then presents a full screen "start" button. The user should be seated straight and tap the screen to start calibration. Calibration runs for 15 seconds to average the orientation values sampled every 0.5 seconds. This helps the application compensate for any misalignment of the phone and/or forward/backwards tilt of the user while seated. While the app is primarily intended for eye-free use, for feedback to the physiotherapist if present a progress bar and time elapsed value is shown screen during calibration (along with accelerometer values to assist with development) (Figure 5 upper). A beep signals the end of the calibration phase when the app automatically starts giving real-time vibrotactile feedback on any side-to-side lean of the user.

The application continuously updates the vibration strength of each motor as the user moves, providing stronger vibration when the user is further from the calibrated center position, and reducing in intensity as they approach an upright position.

Feedback was given on the same side as the lean - so a user leaning left would be given feedback on their left thigh. Again the design intends the user to use the app without looking at the screen but, largely for development purposes, sensor values were shown on screen (Figure 5 lower).

All source code is available under Creative Commons license from

https://mobiquitous.cis.strath.ac.uk/?q=node/377

\section{Data Capture and Initial Testing}

To gain user feedback on how the system is performing and if the vibration feedback is appropriate (e.g. strong and timely enough) we conducted some initial tests with our prototype.

Initial Prototype Expert User Test

Our first data capture test was completed while building the system. This test was run with Dr. Andrew Kerr, a trained physiotherapist working in stroke recovery who was not involved directly in the development of the prototype, and four student participants. This initial test involved testing only the mobile system recording orientation values, with no feedback modules in place. The tests were conducted by video recording and data logging multiple sit-to-stand transitions of straight transition, asymmetrical transitions to both left and right of varying degrees and fast transition by standing up at a faster pace. These motions are based on the range of those most commonly seen amongst stroke rehabilitation patients as determined by Dr. Kerr. These tests allowed for the angle calibration, initial vibration thresholds and differential values to be set and confirmed that the system was able to keep up with the 
speed of motion during the fastest expected transitions. Careful log and video analysis also highlighted some issues with the HTML5 sensor output that were resolved before the final prototype.

\section{Prototype User Tests}

After refining the sensor code and developing the feedback modules, we tested our full prototype with a group of volunteers in order to refine threshold and strength values. Users were volunteers who agreed to take part in the study. While real tests need to be performed with people who are recovering from strokes and have difficulty with sit-to-stand transitions, this group is difficult to work with and the early stage prototype was considered too bulky for trials with these users. As such, the six users were fully-able-bodied students. The study was conducted under institutional ethical approval. Each participant was asked to perform the sit-to-stand transition a number of times (both straight and leaning). After each test users were asked a series of questions for feedback:

- Did you think the threshold angle was suitable?

- Did you think the vibration levels were suitable?

- How comfortable was the system to use?

- How easy was the system to understand?

- How responsive did you find the system to be?

- What would you improve about the product?

These questions were designed in order to gain a large amount of feedback from all of the users, and allowed a judgement to be made on threshold angles and vibration strengths. These tests were used in order to setup the variables for bufferAngle and strength as well as to get feedback on the overall system and how effective users found the feedback.

Prior to these tests the threshold angle, the angle that the user would be required to lean before vibration began, was set to $5^{\circ}$. The vibration strength, a multiplier by which the motor speed increases for each degree past the threshold value the user leans, was also set to $5 x$. After the first few runs were completed with these values set, the feedback suggested that the threshold angle was too high and the vibration strength was too low. Therefore for the second set of tests the threshold angle was set to $2^{\circ}$ and the vibration strength was set at $10 x$. Users were again asked to test the system and provide feedback. This time users said that the threshold angle was suitable. However, when coupled with this lower threshold angle, the vibration strength was now considered too high and that the previously test vibration strength was more suitable. Therefore, the vibration strength was again set to $5 x$ and tests re-run. Feedback now suggested that these values were a suitable combination and that the system provided sufficient feedback that could tell them to stand straighter when they leaned to either side.

Final Prototype Expert User Test

This test was again run with Dr. Kerr and involved him wearing the system in order to judge how effective he felt the feedback system was and how effective it could be in stroke rehabilitation. This allowed him to give expert feedback on how he feels the system would work when used by a stroke rehabilitation patient. This test again involved doing multiple sit-to-stand transitions (straight upwards and leaning on either side to simulate a stroke patient with weakness in one side making the transition). The test was run with the 
threshold value set at $2^{\circ}$ and the vibration strength set at $5 x$, based upon the feedback gained in the previous tests. The feedback was very positive: system

responsive was quick enough and the threshold angle and vibration values were suitable and provided a high level of feedback, particularly the variable proportional vibration levels. This meant that he could continue to adjust his stance until the vibration stopped completely when he reached an upright stance. He suggested that this would provide a nice incentive for users of the system and would provide a confidence boost to recovering patients as they would be able to know that they were improving by reaching an upright stance causing the vibration to stop. Another positive aspect of the feedback received in this test was with the physical independence of the system modules. The expert tester felt that the system was surprisingly comfortable to wear and did not interfere with his sit-to-stand transition or natural movement despite the size of the current prototype leg modules. However, he did feel that the current units were too bulky for patient trials and that miniaturization to some extent was necessary.

\section{Conclusions}

Our prototype has been successful in providing effective and easy to understand dual-leg haptic feedback on sitto-stand operations in real-time. This has been demonstrated as being successful through the use of multiple test cycles, including individual user tests and expert user testing.

Our next stage is to reduce the size of the leg modules. While the equipment performed well, it was considered too bulky to take forward to patient trials. A first step would be to continue to use the Arduino Uno, but to create custom Arduino shields combining the communication and motor control shields. This would reduce the size quite significantly and require very little change in software. A longer term solution would be to design a specialized system on chip system that could be simply plugged in and connected to the vibration motor. This would result in the most compact version of the system albeit at the cost of less flexibility.

Once a miniaturized version is available we plan studies with patients who are undergoing rehabilitation after a stroke.

\section{Acknowledgements}

We thank the volunteers in our studies for their time and effort. We also thank Loraine Clarke and support staff of CIS for their technical help on the project.

\section{References}

1. Bark, K., Hyman, E., Tan, F., et al. Effects of Vibrotactile Feedback on Human Learning of Arm Motions. Neural Systems and Rehabilitation Engineering, IEEE Transactions on 23, 1 (2015), 51-63.

2. Bloomfield, A. and Badler, N. Virtual Training via Vibrotactile Arrays. Presence 17, 2 (2008), 103120.

3. Van Erp, J.B. and Jansen, C. Application of tactile displays in sports: where to, how and when to move. Eurohaptics, (2006).

4. Ganea, R., Paraschiv-Ionescu, A., Büla, C., Rochat, S., and Aminian, K. Multi-parametric evaluation of sit-to-stand and stand-to-sit transitions in elderly people. Medical Engineering \& Physics 33, 9 (2011), 1086 - 1093. 
5. Langhorne, P., Coupar, F., and Pollock, A. Motor recovery after stroke: a systematic review. The Lancet Neurology 8, 8 (2009), 741-754.

6. Nakamura, A., Tabata, S., Ueda, T., Kiyofuji, S., and Kuno, Y. Dance training system with active vibro-devices and a mobile image display. Intelligent Robots and Systems, 2005. (IROS 2005). 2005 IEEE/RSJ International Conference on, (2005), 3075-3080.

7. research2guidance. mHealth App Developer Economics 2014: The State of the Art of mHealth App Publishing. r2guidance, 2014.

8. Spelmezan, D. An Investigation into the Use of Tactile Instructions in Snowboarding. Proceedings of the 14th International Conference on Humancomputer Interaction with Mobile Devices and Services, ACM (2012), 417-426.
9. Spelmezan, D., Jacobs, M., Hilgers, A., and Borchers, J. Tactile Motion Instructions for Physical Activities. Proceedings of the SIGCHI Conference on Human Factors in Computing Systems, ACM (2009), 2243-2252.

10. Uzor, S. and Baillie, L. Exploring \& Designing Tools to Enhance Falls Rehabilitation in the Home. Proceedings of the SIGCHI Conference on Human Factors in Computing Systems, ACM (2013), 1233-1242.

11. Uzor, S., Baillie, L., Skelton, D., and Fairlie, F. Identifying Barriers to Effective User Interaction with Rehabilitation Tools in the Home. In P. Campos, N. Graham, J. Jorge, N. Nunes, P. Palanque and M. Winckler, eds., Human-Computer Interaction - INTERACT 2011. Springer Berlin Heidelberg, 2011, 36-43. 